\title{
Anti-solvent Precipitation Method Coupled Electrospinning Process to Produce Poorly Water-Soluble Drug-Loaded Orodispersible Films
}

Song, Qingchun; Guo, Xiong; Sun, Yi; Yang, Mingshi

\section{Published in:}

AAPS PharmSciTech

Link to article, DOI:

10.1208/s12249-019-1464-2

Publication date:

2019

Document Version

Peer reviewed version

Link back to DTU Orbit

Citation (APA):

Song, Q., Guo, X., Sun, Y., \& Yang, M. (2019). Anti-solvent Precipitation Method Coupled Electrospinning Process to Produce Poorly Water-Soluble Drug-Loaded Orodispersible Films. AAPS PharmSciTech, 20(7), [273]. https://doi.org/10.1208/s12249-019-1464-2

\section{General rights}

Copyright and moral rights for the publications made accessible in the public portal are retained by the authors and/or other copyright owners and it is a condition of accessing publications that users recognise and abide by the legal requirements associated with these rights.

- Users may download and print one copy of any publication from the public portal for the purpose of private study or research.

- You may not further distribute the material or use it for any profit-making activity or commercial gain

- You may freely distribute the URL identifying the publication in the public portal 


\section{Introduction}

2 Tablets and capsules are preferred dosage forms for most patients. However, paediatric,

3 geriatric patients and other patients who suffer from dysphagia or central nervous

4 system diseases may have difficulties in swallowing these dosage forms (1). Over the

5 past two decades, orally disintegrating dosages forms, such as orodispersible tablets

6 (ODTs) and orodispersible films (ODFs), have been developed to circumvent these

7 challenges. Orally disintegrating dosage forms are designed to dissolve or disintegrate

8 rapidly in mouth without usage of water. Most drugs released from these dosage forms

9 are absorbed through gastrointestinal tract, and they may exhibit a rapid onset of action

10 and enhanced bioavailability (2).

There are a few techniques and methods available to produce ODTs, including freeze

13 drying, molding, sublimation, compaction and 3D printing $(3,4)$. Many ODTs launched 14 on the market, e.g. Zofran ${ }^{\circledR}$ and Aricept ${ }^{\circledR}$, were produced by freeze drying and 15 compaction $(5,6)$. One problem with ODTs is their fragility (7). ODFs are relatively new. They are film-like polymeric matrix carrying drug cargo and could be cut into various shapes. ODFs are more flexible and portable as compared to ODTs and they are more precise in dosing than liquid formulations (8). Unlike those mentioned above, the methods for manufacturing ODFs include casting, hot melt extrusion and rolling $(9$, 10). Most of these methods require heating process, so thermal sensitive drugs are normally excluded. Recently, electrospinning has been attempted to prepare oral films because it is versatile and easy to operate $(11,12)$. The diameter of electrospun fibers ranges from nano to micro scales, providing a high surface to volume ratio so as to promote fast dissolving of active substances $(13,14)$.

Electrospun ODFs are generally composed of film forming polymers, plasticizers, saliva simulating agents, and flavouring agents besides active pharmaceutical ingredients (API) $(10,15)$. Among these excipients, film forming polymers constitute a large proportion. Typically, they are water-soluble polymers, such as maltodextrins,

30 hydroxypropyl methylcellulose, pullulan, polyvinyl alcohol and polyvinyl pyrrolidone 31 (16-20). To formulate water-soluble drugs in ODFs, water will be the obvious solvent 32 to dissolve both film forming polymers and APIs (11, 21, 22). However, for poorly 33 water-soluble drugs, it is always necessary to find a common solvent to dissolve both 
hydrophobic/lipophilic APIs and the hydrophilic polymers to form ODFs. As poorly water-soluble drugs are more likely to dissolve in organic solvent, most works used organic solvent for film preparation (23-26). This is a troublesome problem as many water-soluble polymers are not able to dissolve in organic solvent and the massive usage of organic solvent would form an environment burden. Some works directly suspended the poorly water-soluble drugs in aqueous polymer solution to cast films $(17,27)$, while others micronized the drug into micro/nano particles through milling or high-pressure homogenization technique before casting (28-30). Noticeably, the reports about loading micronized poorly water soluble drugs on electrospun ODFs are still rare.

In our work, we investigated the feasibility to combine the anti-solvent precipitation method and the electrospinning process to prepare poorly water-soluble drug loaded ODFs. We proposed to micronize poorly water-soluble drugs in a size range of less than 25 microns, subsequently suspend the micronized drugs in hydrophilic film forming polymer solutions, and then process the suspension into orodispersible fibrous films through electrospinning. The 25 microns of the particle size was chosen mainly for the sake of patient compliance, as the minimum particle size that could be detected by the human palate was 25 microns (31). Piroxicam (PX), a poorly water-soluble drug, catalogued as class II, was chosen as the model drug. The stabilizers and their optimal concentrations for precipitating $\mathrm{PX}$ in anti-solvent precipitation method were selected among poloxamer, copovidone and hydroxypropyl methylcellulose at three different concentrations, respectively. Initial investigations were focused on the development of plain ODF platforms, and therefore, various compositions of PVA and PVP polymer solution were electrospun into films for satisfactory tensile behaviour and disintegration time screen. Polyethylene glycol 300 was added in the formulations to evaluate the influence of plasticizer on the properties of electrospun ODFs. Finally, three PX loaded formulations were prepared, and assessed with various techniques.

\section{Material and Method}

\section{Material}

Piroxicam was purchased from Chr. Olesen Pharmaceuticals A/S (Gentofte, Denmark). Polyvinyl alcohol 26-88 (PVA, Mw 160000) was obtained from Merck (Darmstadt, German) and polyvinylpyrrolidone 360 (PVP, Mw 360000) were purchased from 
Sigma-Aldrich (Schnelldorf, German). Poloxamer (Pluronic F127) was purchased from Sigma-Aldrich (Saint Louis, USA). Polyethylene glycol 300 (PEG300) and hydroxypropyl methylcellulose (HPMC, $15 \mathrm{Cps}$ ) were received from Sigma-Aldrich (Steinheim, German). Copovidone (Kollidon VA64) was purchased from BASF (Ludwigshafen, Germany). Distilled water was used in all experiments. All solvents used were of HPLC grade with the purity $\geqslant 99.8 \%$.

Method

Preparation of PX microcrystal suspensions

PX microcrystal suspensions were prepared with anti-solvent precipitation method. Piroxicam (175 mg) was first dissolved in $2.0 \mathrm{~mL}$ of $\mathrm{N}, \mathrm{N}$ Dimethylformamide (DMF) to make an $8.75 \%(\mathrm{w} / \mathrm{v})$ drug solution. Subsequently, the solution was injected into the $13.0 \mathrm{~mL}$ distilled water containing, either $\mathbf{0 . 2 \%}, \mathbf{0 . 5} \%$ or $1.0 \%$ (w/v) of each stabilizer (i.e. HPMC E15, Kollidon VA64, and Pluronic F127) with a magnet-stirring rate at $1500 \mathrm{rpm}$ in a cold room $\left(4.0^{\circ} \mathrm{C}\right)$.

\section{Particle size measurement}

The particle size distribution of PX microcrystal suspension was measured using a laser diffraction technique with Mastersizer through Hydrosizer 2000S module (Malvern Instruments, Worcestershire, UK). A certain amount of the suspension was dispersed in the sample chamber (distilled water as dispersion medium) at $2500 \mathrm{rpm}$ until the obscuration reached $8 \%-15 \%$. The background was measured for $10 \mathrm{~s}$. The chamber was washed with water after each measurement. The broadness of size distribution (span) was calculated by $\left(\mathrm{D}_{90}-\mathrm{D}_{10}\right) / \mathrm{D}_{50}$, where $\mathrm{d}_{90}$ is defined as the equivalent particle size for which $90 \%$ of the particles are smaller, with definitions of $\mathrm{d}_{50}$ and $\mathrm{d}_{10}$ derived similarly.

A 10\%(w/v) PVA 26-88 was prepared by dissolving 1500mg of the polymer into $15 \mathrm{~mL}$ distilled water under stirring until the polymer was completely dissolved. $A$ 10\%(w/v) PVA/PVP (2/1, w/w) was prepared by dissolving 1000mg of PVA26-88 and 500mg of PVP 360 into $15 \mathrm{~mL}$ distilled water under stirring until the polymers were completely dissolved. Other polymer solutions containing different 

concentration at $10 \%(\mathrm{w} / \mathrm{v})$ with various mass ratios between the two polymers (presented in Table 2 manuscript body). Plain ODFs were obtained by directly electrospinning of the prepared polymer solutions. Drug-loaded ODFs were obtained by dissolving the appropriate amount of film forming polymer into PX microcrystal suspensions produced with anti-solvent method, followed by electrospinning.

After dissolving polymers in PX microcrystal suspensions, the resulting suspensions were kept still in a glass vial for $48 \mathrm{~h}$ in order to evaluate the sedimentation rate of the PX microcrystals in the hydrophilic polymer solutions. At pre-determined time intervals, $10.0 \mu \mathrm{L}$ of the liquid were taken from the top layer of the suspension. The samples were diluted with a binary mixture of water and ethanol $(1: 1, \mathrm{v}: \mathrm{v})$, and analysed

113 through UV analysis.

115 The electrospinning device were customized, equipping with a syringe pump (Pump 11

116 Elite, Harvard Apparatus, Quebec, Canada), a glass syringe (2.5 mL) attached with a 11716 gauge needle $(1.19 \mathrm{~mm}$ inner diameter and $1.65 \mathrm{~mm}$ outer diameter, Hamilton, 118 Bonaduz, Switzerland), a high-voltage supply (PS/FC20R06, Glassman High Voltage,

119 NJ, USA), and a collector wrapped with aluminium foil connected to the ground electrode. The following process parameters were used during electrospinning:

121 electrical high voltage of ca. $9.5-11.5 \mathrm{kV}$, the distance of $10 \mathrm{~cm}$ between the needle and 122 the collector, and the feeding rate of $0.39 \mathrm{~mL} / \mathrm{h}$. The collected ODFs were further dried 123 in a vacuum oven to remove residue solvent and stored in a desiccator for further 124 analyses. The whole setting of the preparation of PX microcrystal suspensions and 125 orodispersible films was illustrated in Figure 1.

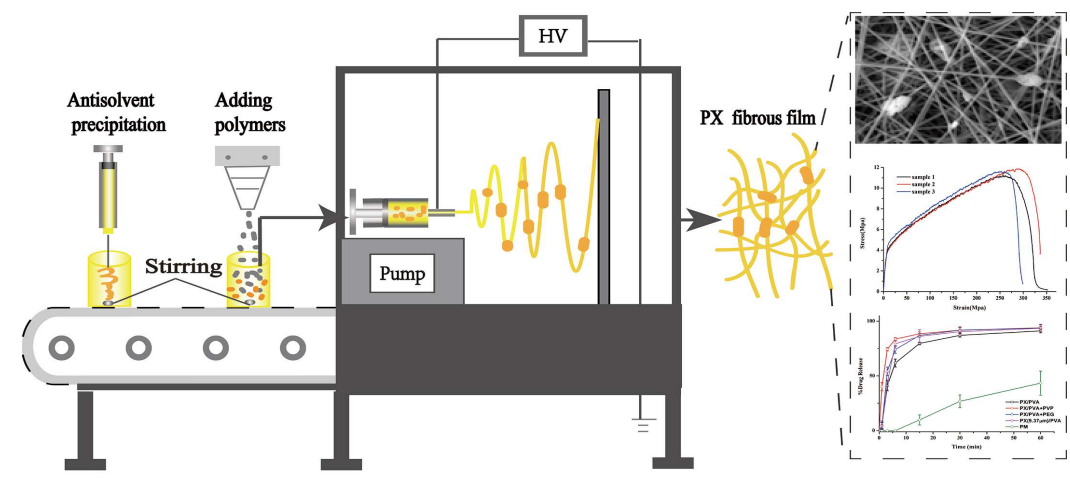




\section{Morphology}

130 The morphology of the samples was observed using a scanning electron microscope

131 (SEM) (Hitachi High-Tech HITACHI, Tokyo, Japan). Prior to observation, samples

132 were stuck on aluminium stubs with double-sided adhesive tape and coated with gold

133 under argon atmosphere. The diameter distribution of the prepared fibers was

134 determined by measuring 200 fibers with the instrument software (TM3030, Hitachi

135 High-Tech HITACHI, Tokyo, Japan).

136

\section{Folding endurance}

138 Folding endurance is a parameter reflecting brittleness. A $2 \times 2 \mathrm{~cm}$ rectangular section

139 of each formulation was repeatedly folded at the same line for 50 times to see whether

140 there was visible damage.

141

\section{Tensile strength}

143 Tensile strength was measured with Dynamic Mechanical Analysis (DMA) (Q800, TA

144 Instruments, New Castle, USA). Samples were cut into 0.4 x $4 \mathrm{~cm}$ rectangular shape

145 with the auxiliary mould. The test mode was DMA Strain Rate with a displacement rate

146 of $200.00 \mu \mathrm{m} / \mathrm{min}$ at room temperature. Prior to tensile strength tests, the thickness of

147 the film was measured using a digital vernier caliper. In brief, three sections from the

148 middle part of the electrospun ODFs were cut for thickness measurement. As fiberous

149 films were porous, certain pressure needed to be applied during measurements. The

150 samples with similar thickness were chosen for the tensile strength measurements.

152 X-ray powder diffraction (XRPD) analysis

153 The X-ray diffractograms of the electrospun ODFs were collected from PANanalytical 154 X'Pert PROMPD X-ray diffractmeter (PANalytical, Almelo, The Netherlands) using $155 \mathrm{CuK} \alpha$ radiation with a wavelength of $1.5418 \AA$. Each sample was put into a zero 156 diffraction plate with a cavity of $10 \mathrm{~mm}$ in diameter and $0.2 \mathrm{~mm}$ in depth. Samples were 157 measured in a spinning mode with a starting angle of $2 \Theta=5^{\circ}$ and an end angle of 
$2 \Theta=35^{\circ}$. The scan speed was $0.6565^{\circ} 2 \Theta / \mathrm{min}$. Data was collected and processed with

159 X'Pert Data Collector (PANalytical, Almelo, The Netherlands)

160

161

162

163

164

165

166

167

168

169

170

171

172

173

174

175

176

177

178

179

180

183

184

185

186

187

\section{Fourier Transform infrared spectroscopy (FTIR)}

Infrared spectra of the electrospun ODFs were collected with Horizon MB 300 FT-IR spectrometer (MB300, ABB Ltd, Zurich, Switzerland) coupled with a MIRacleTM Single Reflection ATR accessory (PIKE Technologies, Fitchburg, USA) to attenuate total reflectance. The scanning range was $4000-400 \mathrm{~cm}^{-1}$ and the resolution was set at $4 \mathrm{~cm}^{-1}$.

\section{Disintegration}

The disintegration of electrospun ODFs was evaluated using a customized method (25, 26). In brief, $2 \times 2 \mathrm{~cm}$ film sections of different formulations were placed into $15 \mathrm{~mL}$ of distilled water in a petri dish with a diameter of $10 \mathrm{~cm}$ at room temperature. The disintegration process was recorded with the video function of a smart phone and the parameter for video recording was $60 \mathrm{fps}$. The disintegration processes of the films were observed afterwards and screenshots were made and reported. The seconds in the results were the time of the screenshots were made.

\section{Drug Loading}

The actual amount of PX was measured by dissolving certain amount of the films in 10 $\mathrm{mL}$ of a mixture of ethanol and water $(1: 1 ; \mathrm{v}: \mathrm{v})$, and analysed through $\mathrm{UV}$ analysis. The drug loading and the entrapment efficiency were calculated through following equations.

$$
\begin{gathered}
\text { Drug loading }(\%)=\frac{\text { Amount of drug in electrospun ODFs }}{\text { Amount of electrospun ODFs }} \times 100 \\
\text { Entrapment efficency }(\%)=\frac{\text { Amount of drug in electrospun ODFs }}{\text { Amount of drug initially added }} \times 100
\end{gathered}
$$



the residue solvent.

\section{In-vitro dissolution study}

192 The dissolution tests were conducted using a USP Apparatus 2 with $250 \mathrm{~mL}$ of

193 dissolution cup and rotating mini paddles (Erweka DT70, Heusenstamm, Germany).

194 Around $12 \mathrm{mg}$ of fiberous films with similar dimension (3 x $3 \mathrm{~cm})$ were cut and

195 immobilized on an aluminium foil in order to ensure that the same surface areas were

196 accessible to the dissolution media. The dissolution media was $150 \mathrm{~mL}$ of distilled

197 water kept at $37 \pm 0.2{ }^{\circ} \mathrm{C}$ and the rotation speed of the paddle was $100 \mathrm{rpm}$. At each

198 predetermined time point, $2 \mathrm{~mL}$ samples were withdrawn and then mixed with $2 \mathrm{~mL}$ of

199 ethanol for UV analysis. After the samples were taken, $2 \mathrm{~mL}$ of fresh dissolution media

200 were added to keep the total volume unchanged.

\section{UV analysis}

203 The quantification of PX in the formulations was analysed using a UV 204 spectrophotometer (Evolution 300, ThermoScientific, Cambridge, UK). In brief, PX 205 standard solutions were prepared by diluting a stock solution $(0.5 \mathrm{mg} / \mathrm{mL}$ PX in 206 ethanol) into the concentrations between $1.0 \mu \mathrm{g} / \mathrm{mL}-5.0 \mu \mathrm{g} / \mathrm{mL}$ with a mixture of 207 ethanol/water (1:1; v:v). The absorption wavelength was selected by scanning the 208 standard solution of $5.0 \mu \mathrm{g} / \mathrm{mL}$ in the UV spectrophotometer. A standard curve was 209 constructed by plotting the concentration of standard solutions as x-axis with its 210 absorbance at $357 \mathrm{~nm}$ as $\mathrm{y}$-axis. The correlation coefficient value (R) was above 0.999 .

211 The experiments were performed at a room temperature.

\section{Statistics}

214 Measurements were performed in triplicate, unless otherwise stated. Results were 215 reported as means \pm S.D. Statistically significant differences were evaluated by one

216 way of an analysis of variance (ANOVA) and t-test using GraphPad Prism version 6 217 (GraphPad Software, San Diego, USA).

219 Results and Discussion

\section{Preparation and characterization of PX suspension}


221 The minimum particle size that could be detected by the human palate was $25 \mu \mathrm{m}(31)$,

222 so the intended particle size of PX microcrystals in the suspension was decided to be 223 less than $25 \mu \mathrm{m}$ for the sake of patients' compliance. Three common polymeric 224 stabilizers, i.e. HPMC E15, Kollidon VA64, and Pluronic F127, were used to prepare 225 PX microcrystals, and the effect of different concentrations of these stabilizers on the 226 particle size distribution of PX microcrystals were studied. It has been reported that the 227 polymers and surfactants adhesive to the drug particles would form steric barrier or 228 electric barrier to prevent the particles growth (32-34). As shown in Table 1, the $\mathrm{D}_{90}$ of 229 the formations containing HPMC were exceeding $30 \mu \mathrm{m}$, whereas the $\mathrm{D}_{90}$ was around $23010-20 \mu \mathrm{m}$ for Kollidon or Pluronic formulations. The $0.5 \%(\mathrm{w} / \mathrm{v})$ and $1.0 \%(\mathrm{w} / \mathrm{v})$ 231 Pluronic formulations had narrower span than the other formulations. In the subsequent 232 studies, the $0.5 \%(\mathrm{w} / \mathrm{v})$ Pluronic formulation was selected for the further studies. 233

234 Table 1 Particle sizes of PX suspensions prepared using different polymeric stabilizers $(\mathrm{n}=3)$.

\begin{tabular}{cccccc}
\hline \multirow{2}{*}{ Material } & Concentration $\%$ & \multicolumn{4}{c}{ Particle Size $(\mu \mathrm{m})$} \\
\cline { 2 - 6 } & $(\mathrm{w} / \mathrm{v})$ & $\mathrm{d}_{10}$ & $\mathrm{~d}_{50}$ & $\mathrm{~d}_{90}$ & Span \\
\hline \multirow{2}{*}{ HPMC } & 0.2 & $7.9 \pm 0.6$ & $16.8 \pm 0.8$ & $31.9 \pm 1.5$ & $1.5 \pm 0.02$ \\
E15 & 0.5 & $9.9 \pm 1.0$ & $21.4 \pm 0.9$ & $39.1 \pm 0.6$ & $1.4 \pm 0.14$ \\
\hline \multirow{2}{*}{ Kollidon } & 1.0 & $7.4 \pm 0.5$ & $18.6 \pm 0.5$ & $36.1 \pm 0.2$ & $1.5 \pm 0.06$ \\
VA64 & 0.2 & $3.5 \pm 0.3$ & $11.6 \pm 0.3$ & $23.5 \pm 0.4$ & $1.7 \pm 0.04$ \\
\hline \multirow{2}{*}{ Pluronic } & 1.0 & $2.7 \pm 0.3$ & $9.4 \pm 0.2$ & $20.3 \pm 0.2$ & $2.0 \pm 0.23$ \\
F127 & 0.5 & $1.7 \pm 0.2$ & $7.4 \pm 0.2$ & $17.7 \pm 0.2$ & $2.2 \pm 0.07$ \\
\hline None & 1.0 & $4.0 \pm 0.2$ & $9.4 \pm 0.2$ & $17.2 \pm 0.4$ & $1.4 \pm 0.02$ \\
Raw PX & - & $3.0 \pm 0.2$ & $6.3 \pm 0.1$ & $10.6 \pm 0.4$ & $1.2 \pm 0.08$ \\
\hline
\end{tabular}

236 Prior to the electrospinning process, the sedimentation rate of the aforementioned PX 237 microcrystals (prepared with $0.5 \%(\mathrm{w} / \mathrm{v})$ Pluronic formulation) in hydrophilic film 238 forming polymer solutions, i.e. PVA and PVA/PVP mixture $(2 / 1 ; \mathrm{w} / \mathrm{w})$ were 239 investigated. As shown in Figure 2, no obvious sedimentation of PX microcrystals 240 occurred within the first 5 hours. This could be attributed to the viscous nature of the 
241 film forming polymer solutions. In the subsequent electrospinning process, $1.5 \mathrm{~mL}$ of 242 the feed was electrospun at a flow rate of $6.5 \mu \mathrm{L} / \mathrm{min}$. The course of the electrospinning 243 process was ca. $3.9 \mathrm{~h}$. This implied that no obvious sedimentation of PX microcrystals 244 would occur during the electrospinning process, which may ensure the uniformity of 245 the dosage forms.

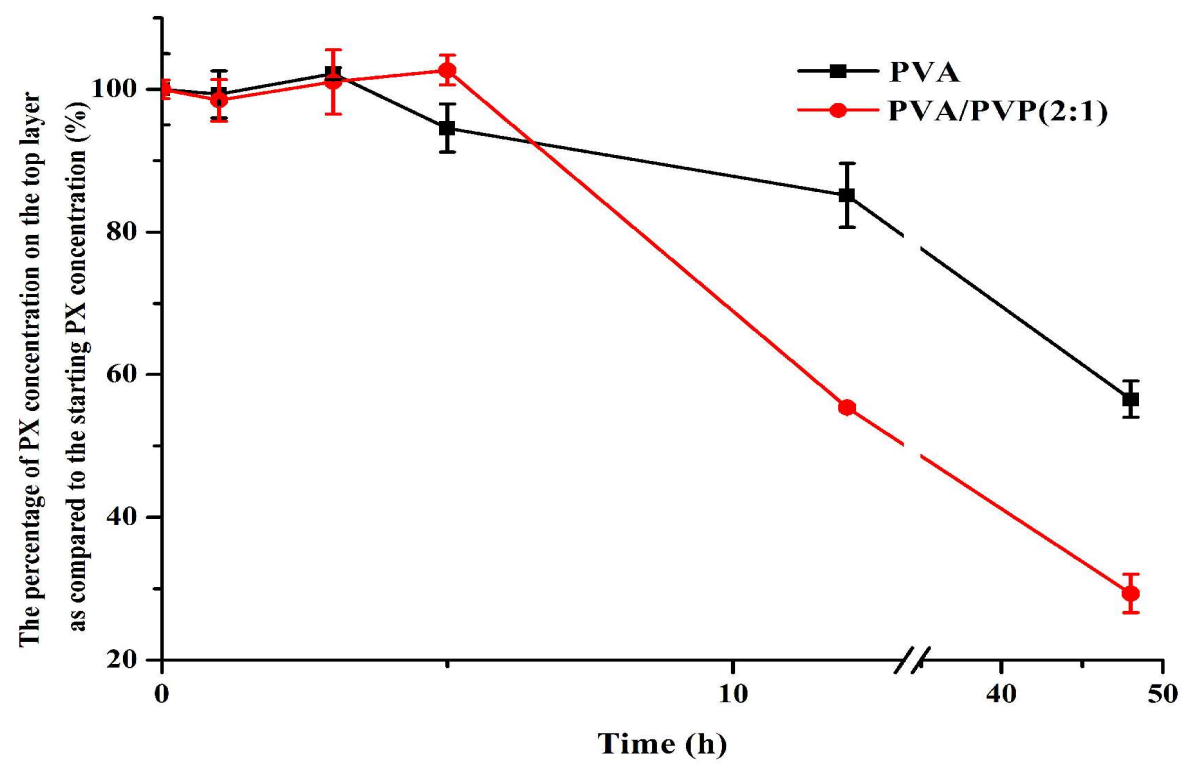

246

Fig. 2 Sedimentation rate of PX suspensions in film forming polymer solutions $(\mathrm{n}=3)$.

\section{Preparation and characterization of plain electrospun ODFs}

250 Initially, many polymer solutions (Table 2) were electrospun into films to construct a 251 suitable film platform for loading PX microcrystals. As shown in Table 2, with more 252 PVP in films, both the tensile strength and disintegration time dropped profoundly. The 253 mixture of PVA/PVP at mass ratio of 2/1 was selected for the following study due to 254 its short disintegration time without compromising the tensile strength much.

255

256 Table 2 Tensile strength and disintegration time of films containing PVA/PVP at various ratios 257

\begin{tabular}{ccc} 
& $(\mathrm{n}=3)$. & \\
\hline $\mathrm{PVA} / \mathrm{PVP}(\mathrm{w} / \mathrm{w})$ & Tensile strength $(\mathrm{Mpa})$ & Disintegration time(s) \\
\hline $1 / 0$ & $14.5 \pm 0.9$ & $\sim 180$ \\
\hline
\end{tabular}




\begin{tabular}{lll}
\hline $4 / 1$ & $12.5 \pm 1.0^{*}$ & $\sim 25$ \\
$2 / 1$ & $12.0 \pm 0.8^{*}$ & $\sim 5$ \\
$1 / 1$ & $10.0 \pm 0.4 * * *$ & $<1$
\end{tabular}

258 Levels of significance are $* \mathrm{P}<0.05$ as compared to the films prepared from pure $\mathrm{PVA} ; * * * \mathrm{P}<0.001$ as

Table 3. Formulation composition of electrospun ODFs with and without loading of PX.

\begin{tabular}{|c|c|c|c|c|c|c|}
\hline \multirow[t]{3}{*}{ No. } & \multirow[t]{3}{*}{ Code } & \multicolumn{5}{|c|}{ Ingredient (mg) } \\
\hline & & \multirow{2}{*}{$\begin{array}{l}\text { API } \\
\text { PX }\end{array}$} & \multirow{2}{*}{$\begin{array}{c}\text { Stabilizer } \\
\text { Pluronic F127 }\end{array}$} & \multicolumn{2}{|c|}{ Film forming polymers } & \multirow{2}{*}{$\begin{array}{l}\text { Plasticize } \\
\text { PEG300 }\end{array}$} \\
\hline & & & & PVA26-88 & PVP360 & \\
\hline 1 & PVA & 0 & 0 & 1500 & 0 & 0 \\
\hline 2 & PX/PVA & 175 & 65 & 1500 & 0 & 0 \\
\hline 3 & $\mathbf{P X} / \mathbf{P V A}+\mathbf{P V P}$ & 175 & 65 & 1000 & 500 & 0 \\
\hline 4 & PX/PVA+PEG & 175 & 65 & 1500 & 0 & 56.5 \\
\hline
\end{tabular}

\section{Preparation of PX loaded electrospun ODFs}

264 Three PX microcrystal loaded ODFs were prepared (Table 3). The total concentration

265 of electrospun polymers solutions was kept at 10\% (w/v). The ODFs without PX was

266 also produced as a reference group. These formulations were successfully electrospun

267 into fiberous films. The plain films without PX were white, while films containing PX

268 were yellow by visual inspection. Besides PVP and PVA, an addition of PEG300 as a

269 plasticizer was attempted to investigate its effect on the physicochemical properties of 270 the ODFs. 
274 As seen from the SEM images in Figure 3, the diameters of most electrospun fibers

275 were under micron size. There were some particles on the surface or entrapped between 276 the fibers in PX loaded films, but not in the reference PVA films, which were most 277 likely due to the PX microcrystals. The mean diameters of the fibers in the PX/PVA 278 film were $290 \pm 58 \mathrm{~nm}$. When PVP was added, the mean diameters of the fibers in film 279 were decreased to $206 \pm 55 \mathrm{~nm}$, while the mean diameter of the fibers in the film 280 containing PEG 300 was increased to $415 \pm 58 \mathrm{~nm}$. The possible reasons for diameter 281 changing could be the different rheology properties of the polymer solutions in various 282 compositions. Some researchers also reported that the diameters of electrospun fibers 283 changed with an addition of drugs and varying excipients (35-37).

284

285
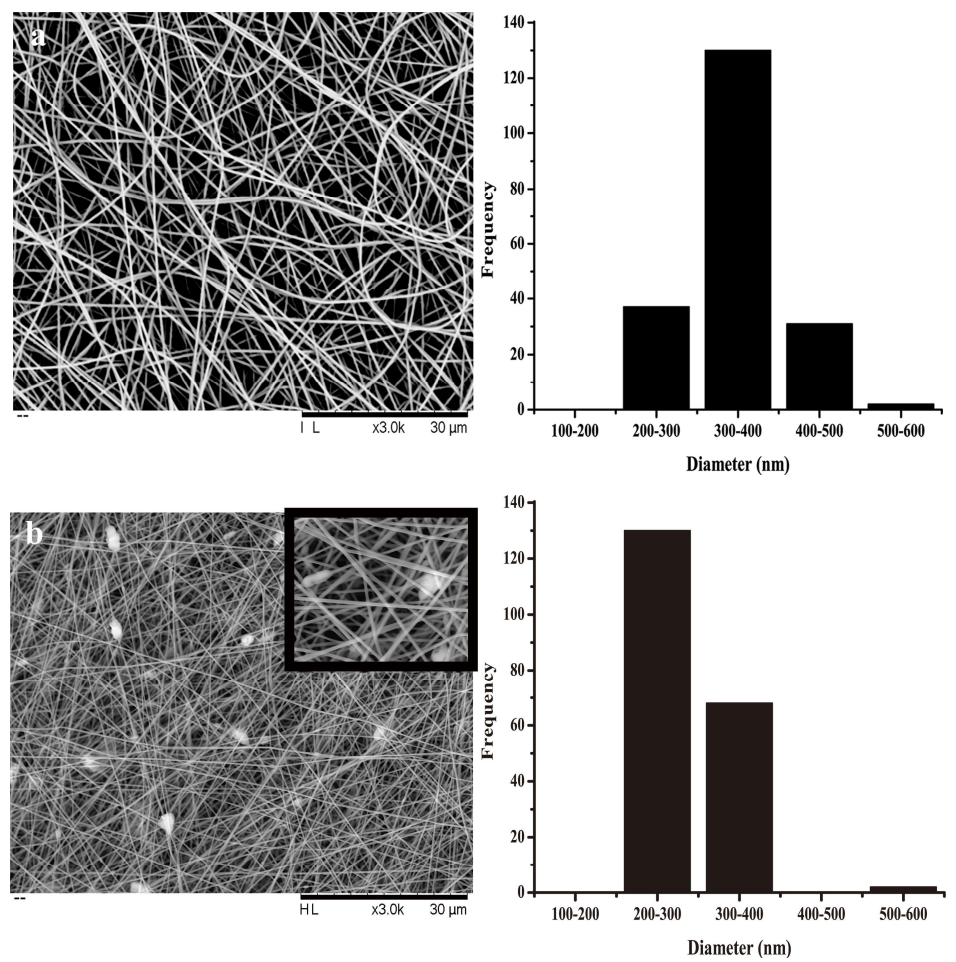

286
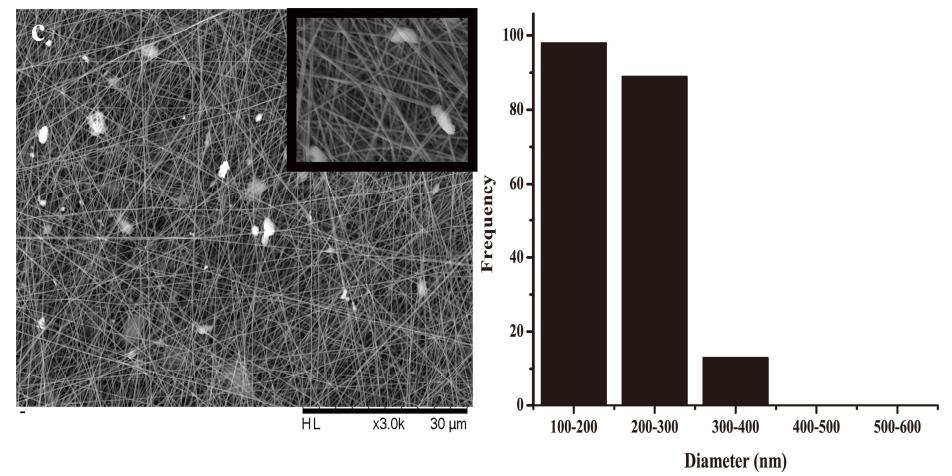

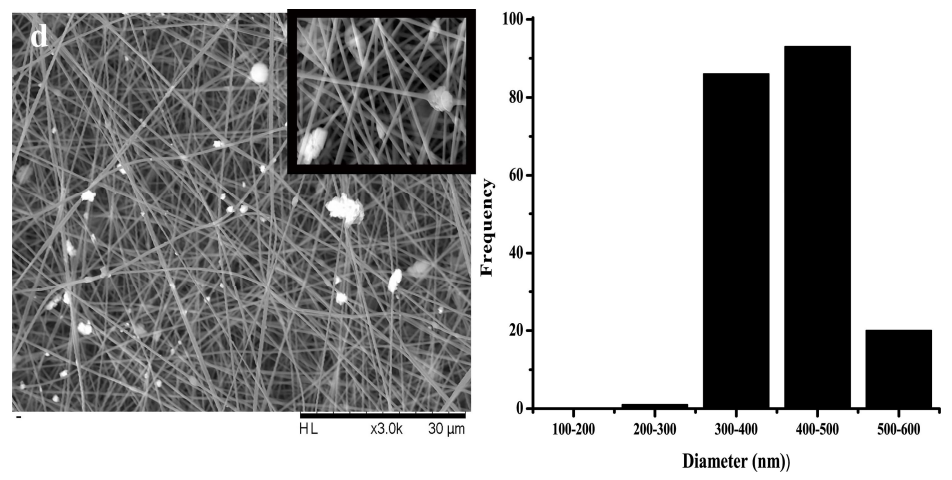

Fig.3 SEM images of PVA (a); PX/PVA (b); PX/PVA+PVP (c); PX/PVA+PEG (d) films and its corresponding diameter distribution.

\section{Mechanical properties}

ODFs are expected to possess certain mechanical properties to prevent them from damage during handling and transportation. In general, the tensile strength and folding endurance of ODFs are two vital parameters to assess the mechanical properties of ODFs. The curves in Fig. 4 showed that the electrospun ODFs exhibited typical stressstrain behaviours of plastic materials with ductility. It contained a short elastic phase that the strain increased in the proportion with stress and a long plastic phase when the film could no longer return to its original shape. All formulations showed similar curve patterns exhibiting the characteristic of ductility of the films (Fig. 4a). It was obvious from Fig. $4 \mathrm{~b}$ that the films containing PVP were unlikely to deform (less strain with the same stress) which would be a better product as compared to others with regard to film handling.
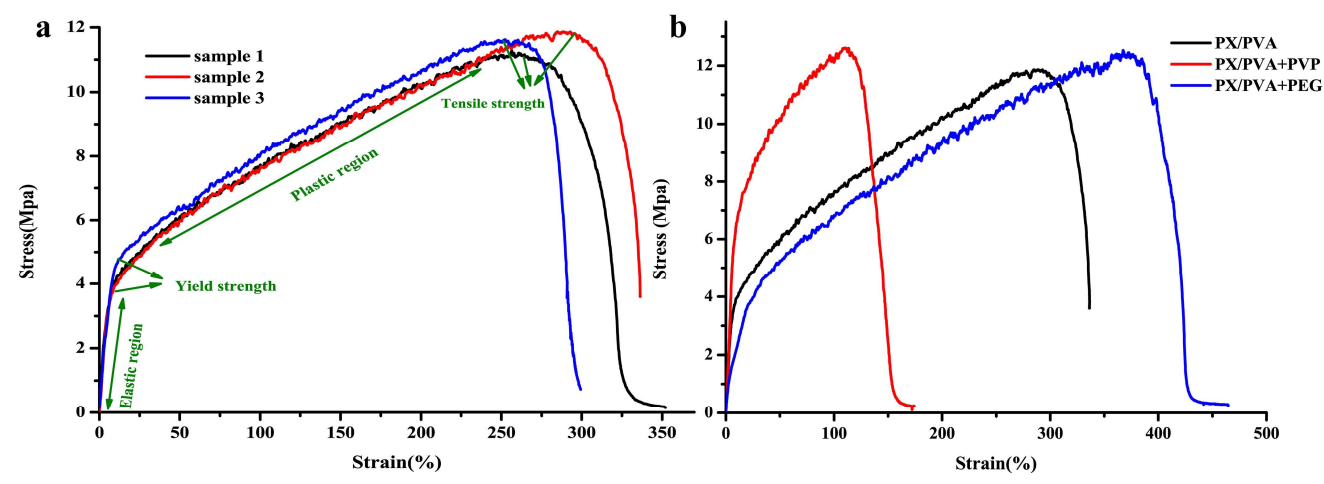

Fig.4 Stress-strain curves of PX/PVA films in three measurements (a); Representative stress-strain curves of three PX loaded formulations (b). 
309 Based on the curves, three indexes were calculated, namely Young's modulus (an index 310 of stiffness), tensile strength and yield strength. The plain PVA films had significant 311 higher Young's modulus $108.3 \pm 22.1 \mathrm{MPa}$ than the PX microcrystals loaded 312 electrospun ODFs (Fig.5). It implied that an addition of PX microparticles in PVA 313 films compromised the stiffness. This may be because the addition of PX microparticles 314 disrupted the continuation of fibers and their arrangements, and thus weakened the 315 intermolecular strength of the fibers.

The PX/PVA+PVP films exhibited significant higher yield strength among all PX 318 loaded formulations showing superior ability to keep original shapes. It showed that an addition of PEG300 as the plasticizer in the film formulations resulted in a decrease in Young's modulus and yield strength, but was not statistically significant from the other two formulations. Interestingly, the mean tensile strengths of the three electrospun ODFs were similar. It was probably due to the fact that PVA constituted a large proportion of all formulations, and therefore, tensile strength, the maximum tension that the films could withstand before breaking, was mainly depended on the PVA mechanical property. All the formulations could be folded more than 50 times without break.

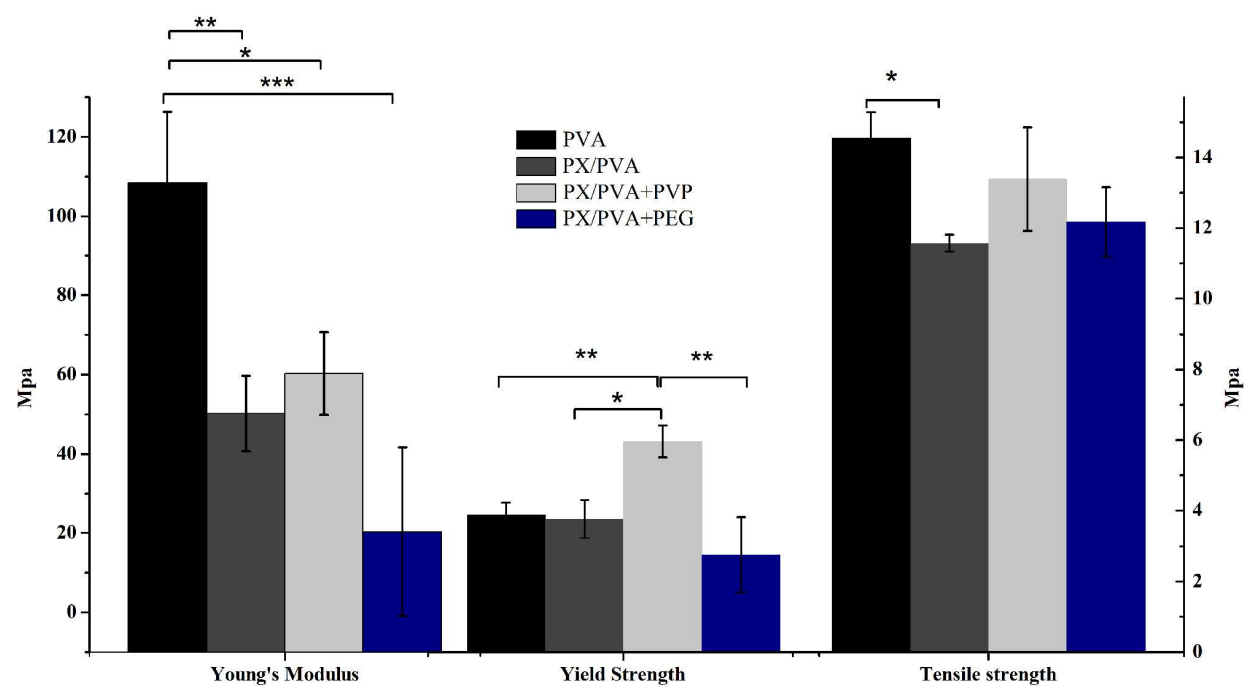

Fig.5 Mechanical properties of plain PVA film and PX loaded films, ${ }^{*} \mathrm{P}<0.05,{ }^{* *} \mathrm{P}<0.01,{ }^{* * *} \mathrm{P}<0.001$. 
332 PX was reported to have four polymorphic forms, namely Form I, II- $\alpha 1$, II- $\alpha 2$, III and 333 monohydrate form (38). As shown in the XRPD diffractgrams in Fig.6a, the starting 334 material, bulk PX powder, had intense diffractions at $8.93^{\circ}, 18.06^{\circ}$ and $27.08^{\circ}(2 \theta)$, 335 which were the characteristic diffractions of Form I. The PX microcrystals obtained 336 from the anti-solvent precipitation method was upper-concentrated by centrifugation 337 and analysed immediately with the X-ray diffractometer. The characteristic diffraction 338 of this sample at around $26.61^{\circ}$ (Fig. 6b) suggested that it was in the monohydrate form 339 (39). These indicated that PX transformed from Form I to monohydrate form after anti-solvent precipitation. The physical mixtures were prepared with monohydrate form PX and the polymers in the same ratios as in the drug-loaded ODFs. Comparing the XRPD patterns of the physical mixtures with their corresponding formulation diffractgrams, PX microparticles kept their monohydrate form, which suggested that the electrospinning process did not distort the solid state of PX microparticles.

$\mathbf{a}$

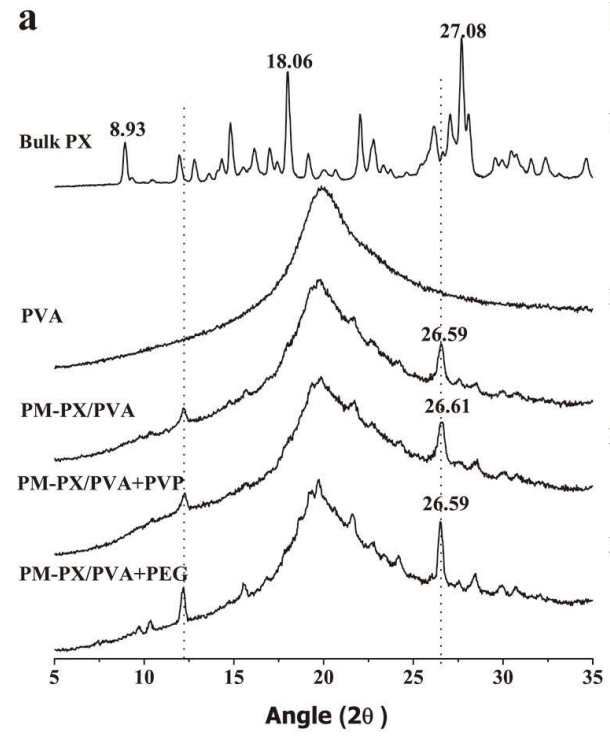

b

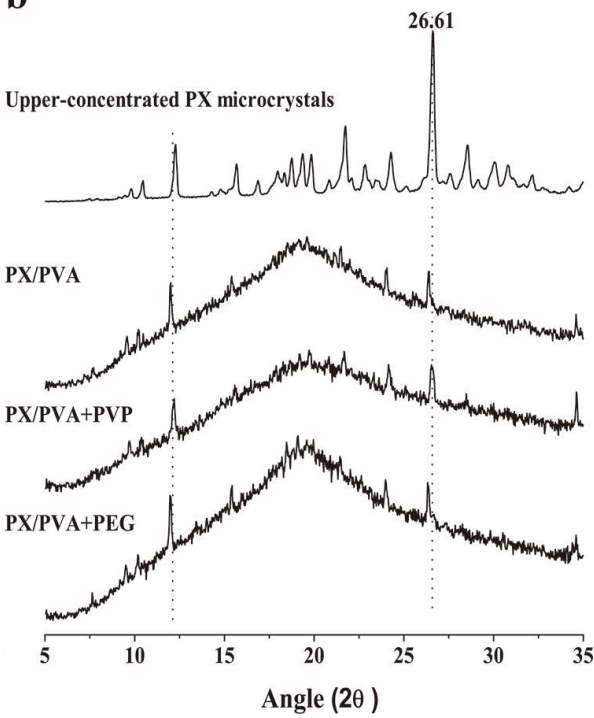

Fig. 6 Representative XRPD patterns of bulk PX, ball-milled PVA, and physical mixtures of monohydrate form PX and polymers (a); Representative XRPD patterns of d upper-concentrated PX microparticles, and the three formulations (b).

To investigate potential molecular interactions between PX and the excipients, the films were subjected to FTIR. The FTIR spectrum of PX in monohydrate form was used as 
354 a reference. The infrared spectrum of PX monohydrate form had the characteristic 355 peaks at $1641 \mathrm{~cm}^{-1}$ of $\mathrm{C}=\mathrm{O}$ stretching and $1326 \mathrm{~cm}^{-1}$ of symmetrical $\mathrm{O}=\mathrm{S}=\mathrm{O}$ stretching 356 (Fig. 7a). The spectrum of PVP powder showed C-H stretch between 2826-3023 $\mathrm{cm}^{-1}$, $357 \mathrm{C}-\mathrm{N}$ stretch at $1265 \mathrm{~cm}^{-1}$ and $\mathrm{C}=\mathrm{O}$ at $1654 \mathrm{~cm}^{-1}$, while the very broad band at 3650 $3583150 \mathrm{~cm}^{-1}$ could be assigned to O-H stretching from water absorbed by PVP (40). 359 Similarly, The bands between $2920 \mathrm{~cm}^{-1}-2940 \mathrm{~cm}^{-1}$ in PVA spectrum were attributed 360 to $-\mathrm{CH}_{2}$ - and $-\mathrm{CH}_{3}$ - stretching (Fig. 7a). The peak at $1092 \mathrm{~cm}^{-1}$ in PVA was $\mathrm{C}-\mathrm{O}$ 361 stretching. The very broad band at around $3319 \mathrm{~cm}^{-1}$ was partly attributed to hydroxyl 362 group in PVA structure and partly because of water absorption. In spectrum of PX 363 loaded films, most of PX characteristic signals were overlapped with the polymer 364 signals (Fig.7b). The main functional groups of polymers remained at the same place 365 in Fig.7, suggesting no molecular interaction between PX and polymers occurred.

367

368 369

370

371

372 373 374 375 376
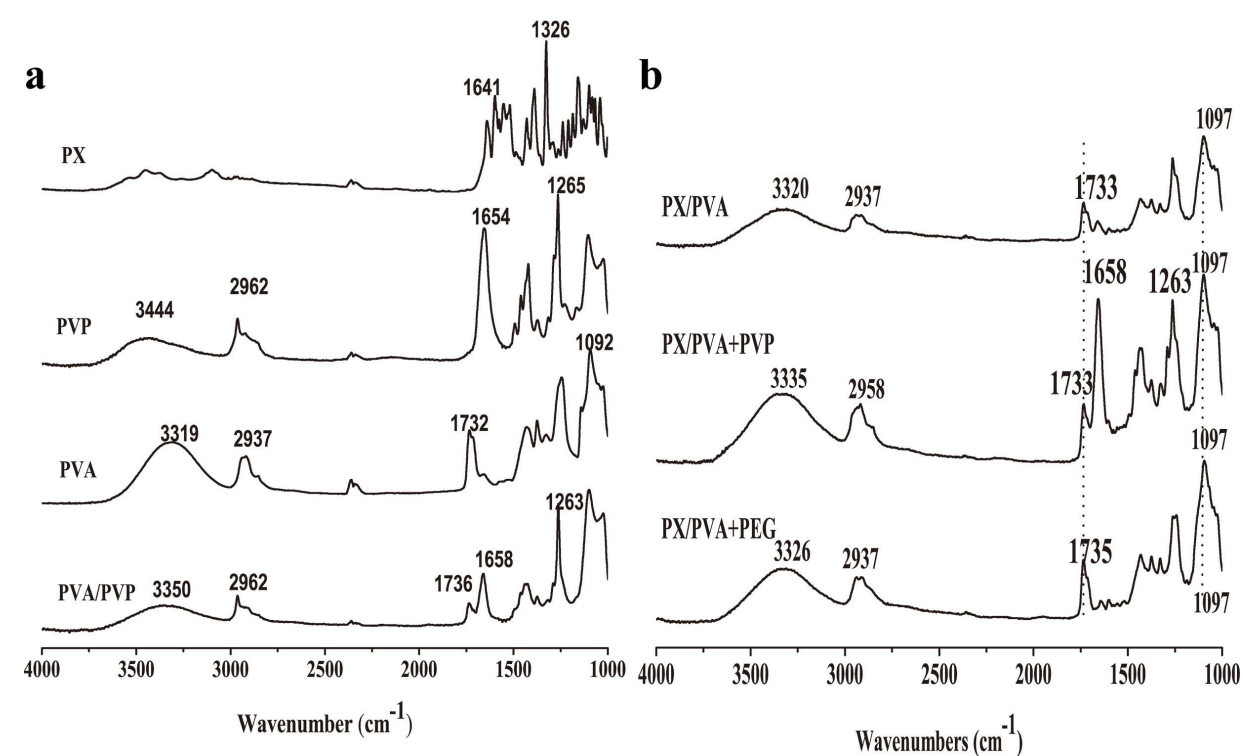

Fig.7 FT-IR spectra of bulk materials (a) and the three formulations (b).

\section{Disintegration}

Disintegration time is one of the key quality attributes of ODFs. The disintegration of the electrospun ODFs ranged from a few seconds to 200 seconds depending on the formulation compositions. During the disintegration process, PX/PVA films first got wet, and then shrank to gel-like small pieces. Eventually, the film disappeared within 200 seconds. The similar phenomenon was also observed for PX/PVA+PEG films. In contrast, PX/PVA+PVP films rapidly disintegrated into small pieces when contacted 
377 with water. It exhibited much shorter disintegration time ( $3 \mathrm{~s})$ as compared to the other 378 two formulations. The shorter disintegration time of PX/PVA+PVP films may be 379 because that PVP dissolved faster than PVA in water. It has been reported that the 380 disintegration of PVP films usually was very fast, however, the disintegration time 381 tended to be prolonged, when other excipients such as PVA were formulated $(41,42)$. 382 The screen shots of the disintegration of various films were presented in Table 4. As 383 shown, the electrospun ODFs disappeared by the time of disintegration.

Table 4 Disintegration times of three formulations $(n=3)$.

Disintegration Time (s) Disintegration process

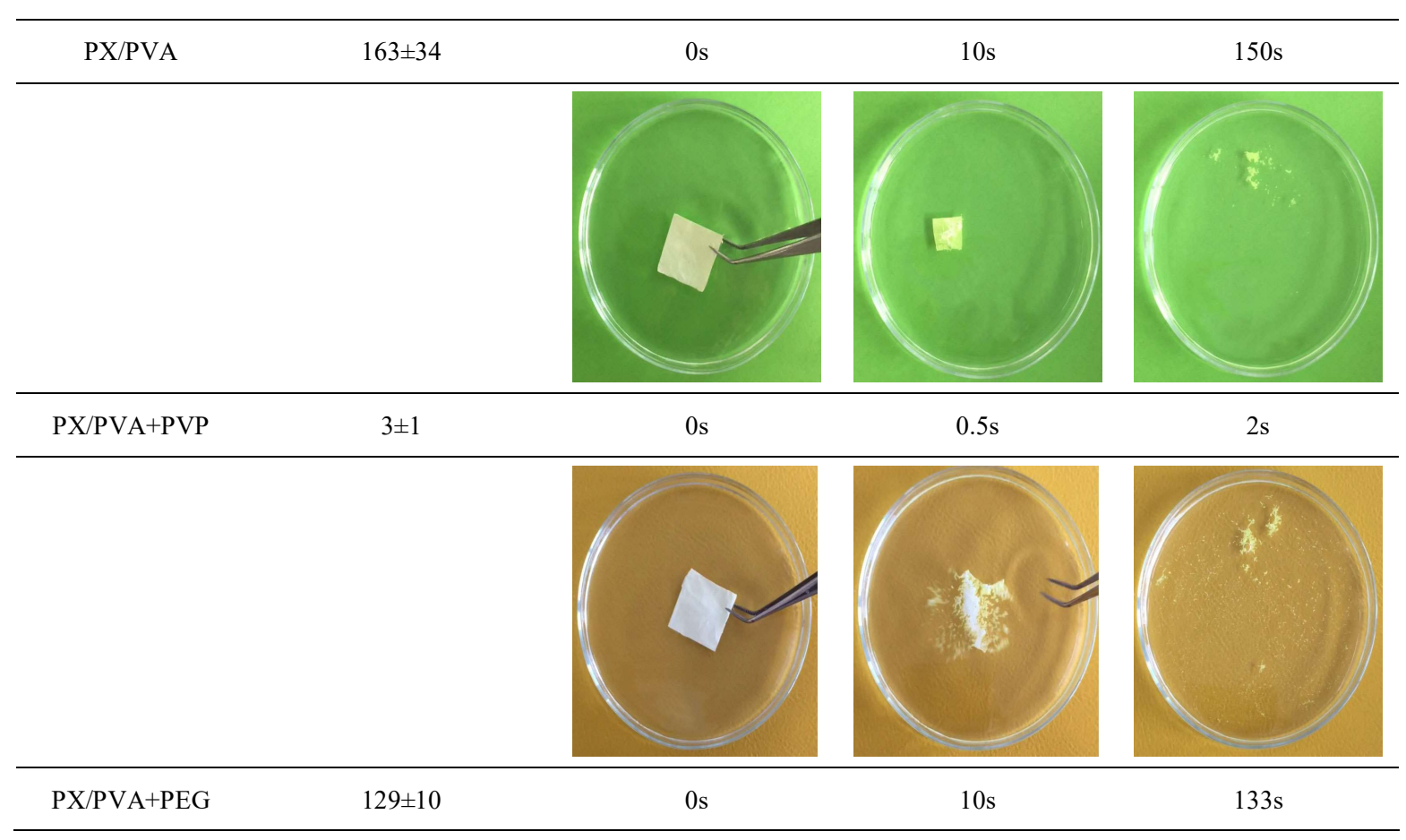




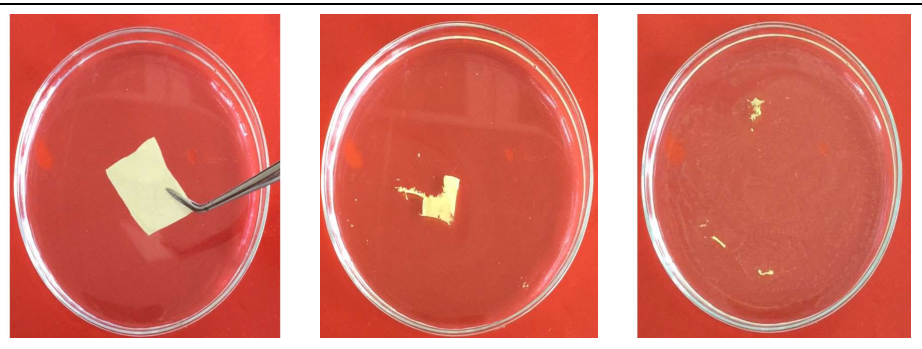

391

392 Drug loading and Residue solvent

393 Prior to dissolution tests, the drug loading and entrapment efficiency of the films were

394 calculated and listed in Table 5. There was significant difference in drug loading

395 between PX/PVA and PX/PVA+PEG films. This may be attributed to the instability of

396 the electrospinning process with PX/PVA+PEG formulation. In fact, it was observed

397 that the addition of PEG300 as a plasticizer greatly disturbed the electrospinning

398 process. In addition, the entrapment efficiency of PX in the PX/PVA+PEG films was

399 significant lower than PX/PVA and PX/PVA+PVP films.

400

401

402

403

Table 5. Characterizing data for electrospun ODFs $(n=3)$.

\begin{tabular}{cccccc}
\hline Formulation & $\begin{array}{c}\text { Drug load } \\
(\%)\end{array}$ & $\begin{array}{c}\text { Entrapment efficiency } \\
(\%)\end{array}$ & $\begin{array}{c}\text { Residue solvent } \\
(\%)\end{array}$ & $\begin{array}{c}\text { Thickness } \\
(\mu \mathrm{m})\end{array}$ & $\begin{array}{c}\text { Weight } \\
(\mathrm{mg})\end{array}$ \\
\hline PX/PVA & $8.88 \pm 0.26$ & $88.25 \pm 2.59$ & $3.8 \pm 0.2$ & $43.0 \pm 4.0$ & $11.5 \pm 0.2$ \\
PX/PVA+PVP & $8.80 \pm 0.50$ & $87.51 \pm 5.00$ & $3.4 \pm 0.1$ & $45.0 \pm 1.0$ & $12.8 \pm 0.1$ \\
PX/PVA+PEG & $7.94 \pm 0.29 *$ & $81.54 \pm 2.95$ & $3.1 \pm 0.4$ & $46.0 \pm 3.1$ & $12.5 \pm 0.4$ \\
\hline
\end{tabular}

404

Levels of significance are $* \mathrm{P}<0.05$ as compared to drug loading of PX/PVA films.

405

406 DMF was used as one of the solvents for electrospun films preparation and it 407 constituted $12.6 \%(\mathrm{w} / \mathrm{v})$ in the initial PX microcrystal suspension. To measure the 408 percentage of the residue solvent, the obtained ODFs were subjected to TGA 409 measurement. The weight loss until $180^{\circ} \mathrm{C}$ was calculated for residue solvent (the 410 boiling point of DMF is $153^{\circ} \mathrm{C}$ ). It was found that most of the weight loss occurred 411 before $100{ }^{\circ} \mathrm{C}$ and the rate of weight loss became slow between $100{ }^{\circ} \mathrm{C}$ and $160{ }^{\circ} \mathrm{C}$. 412 After $180{ }^{\circ} \mathrm{C}$ a dramatic weight loss was observed, which was assigned to 
413 decomposition of the chemicals. The results showed that the residue solvents of the

414 three formulations were between 3\%-4\% (Table 5).

415

416

417 In-vitro dissolution profile

418 Different in-vitro dissolution methods were reported to test ODFs, such as small

419 volume of media (mimic oral cavity) in container coupled with magnet stirring $(25,26)$

420 and shaker incubator (42). The method used in this study was traditional paddle method

421 (Erweka apparatus) as our EEFs based ODFs were designed to disintegrate or dissolve

422 fast in mouth for ease of swallowing, and the dissolved PX was intended to be absorbed

423 through gastrointestinal tract.

424

425 The thicknesses of the film cuts for dissolution study were measured and the 426 corresponding weights of the films were presented in Table 5. As shown in Figure

4278 , the dissolution rates of the three electrospun ODFs were faster than that of the 428 physical mixture (PM). Within the first 15 minutes, $79.7 \pm 1.4 \%, 88.0 \pm 1.4 \%$ and 87.1

$429 \pm 2.0 \%$ of PX were dissolved from the PX/PVA, PX/PVA+PVP and PX/PVA+PEG 430 films, respectively, whereas only $9.8 \% \pm 5.7 \%$ of $\mathrm{PX}$ was dissolved from the physical 431 mixture of PVA and PX. In addition, PX in the films composed of PVA and PVP 432 dissolved faster than the films composed of only PVA or PVA+PEG. These results 433 were in consistent with the disintegration study, where PX/PVA+PVP film 434 disintegrated much faster than PX/PVA film. PEG300 demonstrated no obvious effects 435 on PX dissolution profile, as shown in Figure 8.

436 


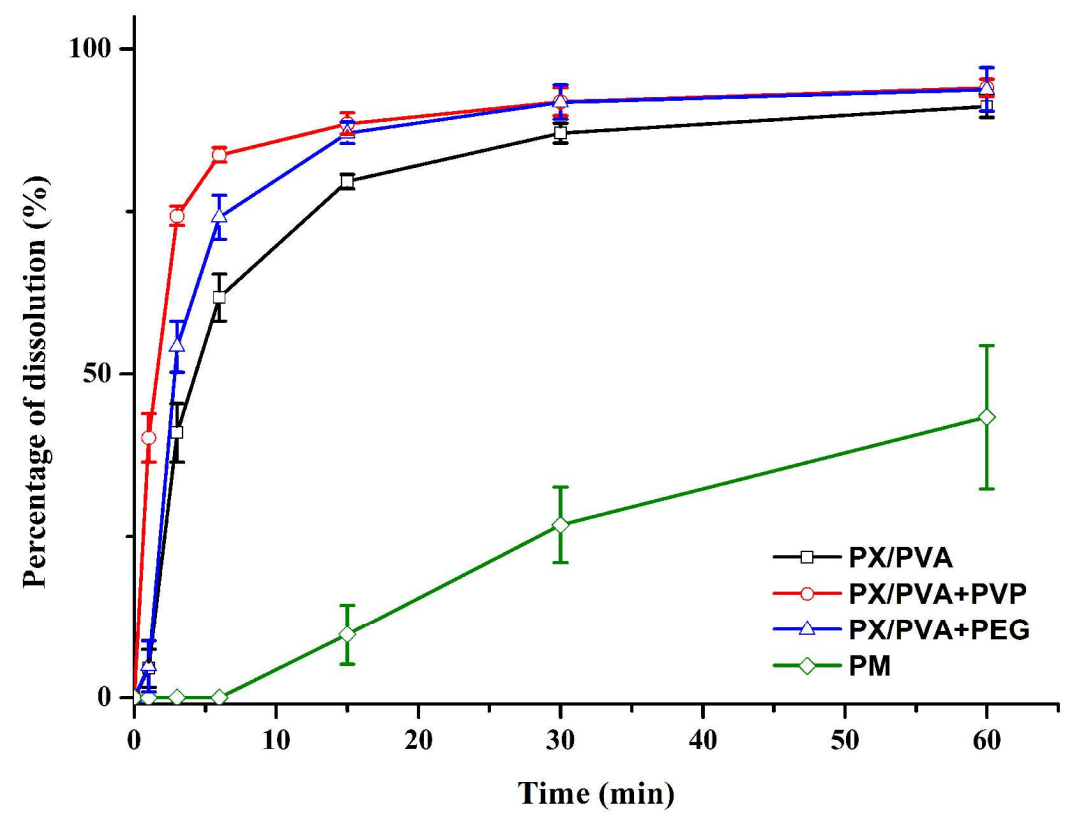

Fig. 8 Dissolution profiles of PX-loaded electrospun ODFs and the physical mixture (PM) of PX/PVA.

440 Compared to the physical mixture of PX and PVA, the faster dissolution rate of PX in 441 electrospun ODFs can be attributed to the high surface area of PX microcrystals as 442 compared to the bulk powder. Size reduction by the anti-solvent precipitation method 443 enlarged the surface area of PX, thereby improved dissolution rate as illustrated by 444 Noyes-Whitney equation (43). Moreover, as shown in XRPD results (Fig.6), PX in the 445 formulations was monohydrate, while raw API was form I. PX in different crystalline 446 forms exhibited distinct dissolution rates (44), with the highest value for monohydrate, 447 followed by form II, form III and form I. In addition, as electrospun ODFs were porous 448 with high surface area, the PX microparticles entrapped between the fibers was more 449 likely to get access to dissolution media. Finally, the hydrophilic excipients, PVA, PVP 450 and PEG 300 in the formulation could not only contribute to fast disintegration and 451 dissolution of films, but also facilitate wetting of the particles.

\section{Conclusion}

454 In this study, piroxicam, a poorly water-soluble model drug, was loaded into ODFs by 455 electrospinning of micronized piroxicam praticles obtained from the anti-solvent 456 precipitation method. The electrospun orodispersible films exhibited satisfactory 
457 mechanical properties, folding endurance and in-vitro disintegration performance. This

458 study demonstrated the feasibility of formulating poor water-soluble drug into ODFs

459 without using massive organic solvents by coupling the electrospinning process with

460 the anti-solvent precipitation method.

461

462

463

\section{References}

464 1. Bhosle M, Benner JS, Dekoven M, Shelton J. Difficult to swallow: patient 465 preferences for alternative valproate pharmaceutical formulations. Patient 466 preference and adherence. 2009;3:161-71.

467 2. Borges AF, Silva C, Coelho JF, Simoes S. Oral films: Current status and future 468 perspectives: I - Galenical development and quality attributes. Journal of controlled 469 release : official journal of the Controlled Release Society. 2015;206:1-19. doi: $470 \quad 10.1016 /$ j.jconrel.2015.03.006.

471 3. Hirani JJ, Rathod DA, Vadalia KR. Orally disintegrating tablets: a review. 472 Tropical journal of pharmaceutical research. 2009;8(2).

473 4. Douroumis D. Orally disintegrating dosage forms and taste-masking 474 technologies; 2010. Expert Opin Drug Deliv. 2011;8(5):665-75. doi: $475 \quad 10.1517 / 17425247.2011 .566553$.

476 5. Venkatesh GM. Ondansetron Orally Disintegrating Tablet Compositions for 477 Prevention of Nausea and Vomiting. Google Patents; 2011.

478 6. Pilgaonkar P, Rustomjee M, Gandhi A, Bagde PM. Orally disintegrating tablets. 479 Google Patents; 2013.

480 7. Elnaggar YS, El-Massik MA, Abdallah OY, Ebian AE. Maltodextrin: a novel 481 excipient used in sugar-based orally disintegrating tablets and phase transition 482 process. AAPS PharmSciTech. 2010;11(2):645-51. doi: 10.1208/s12249-010-9423483 y.

484 8. Arya A, Chandra A, Sharma V, Pathak K. Fast dissolving oral films: an 485 innovative drug delivery system and dosage form. International Journal of Chem 486 Tech Research. 2010;2(1):576-83.

487 9. Repka MA, Gutta K, Prodduturi S, Munjal M, Stodghill SP. Characterization of 488 cellulosic hot-melt extruded films containing lidocaine. European journal of 489 pharmaceutics and biopharmaceutics : official journal of Arbeitsgemeinschaft fur 
490 Pharmazeutische Verfahrenstechnik eV. 2005;59(1):189-96. doi:

491 10.1016/j.ejpb.2004.06.008.

492 10. Irfan M, Rabel S, Bukhtar Q, Qadir MI, Jabeen F, Khan A. Orally disintegrating

493 films: A modern expansion in drug delivery system. Saudi pharmaceutical journal :

494 SPJ : the official publication of the Saudi Pharmaceutical Society. 2016;24(5):537-

495 46. doi: 10.1016/j.jsps.2015.02.024.

496 11. Vuddanda PR, Mathew AP, Velaga S. Electrospun nanofiber mats for ultrafast

497 release of ondansetron. Reactive and Functional Polymers. 2016;99:65-72. doi:

$498 \quad 10.1016 /$ j.reactfunctpolym.2015.12.009.

499 12. Krstic M, Radojevic M, Stojanovic D, Radojevic V, Uskokovic P, Ibric S.

500 Formulation and characterization of nanofibers and films with carvedilol prepared

501 by electrospinning and solution casting method. European journal of pharmaceutical

502 sciences : official journal of the European Federation for Pharmaceutical Sciences.

503 2017;101:160-6. doi: 10.1016/j.ejps.2017.02.006.

504 13. Yu DG, Yang JM, Branford-White C, Lu P, Zhang L, Zhu LM. Third generation

505 solid dispersions of ferulic acid in electrospun composite nanofibers. Int J Pharm.

506 2010;400(1-2):158-64. doi: 10.1016/j.ijpharm.2010.08.010.

507 14. Wang Q, Yu DG, Zhang LL, Liu XK, Deng YC, Zhao M. Electrospun

508 hypromellose-based hydrophilic composites for rapid dissolution of poorly water-

509 soluble drug. Carbohydr Polym. 2017;174:617-25. doi:

$510 \quad 10.1016 /$ j.carbpol.2017.06.075.

511 15. Sakellariou P, Rowe R. Interactions in cellulose derivative films for oral drug

512 delivery. Progress in polymer science. 1995;20(5):889-942.

513 16. Mahesh A, Shastri N, Sadanandam M. Development of taste masked fast

514 disintegrating films of levocetirizine dihydrochloride for oral use. Current drug 515 delivery. 2010;7(1):21-7.

516 17. Cilurzo F, Cupone IE, Minghetti P, Selmin F, Montanari L. Fast dissolving films 517 made of maltodextrins. European journal of pharmaceutics and biopharmaceutics :

518 official journal of Arbeitsgemeinschaft fur Pharmazeutische Verfahrenstechnik eV.

519 2008;70(3):895-900. doi: 10.1016/j.ejpb.2008.06.032.

520 18. Kunte S, Tandale P. Fast dissolving strips: A novel approach for the delivery of 521 verapamil. Journal of pharmacy \& bioallied sciences. 2010;2(4):325-8. doi: $52210.4103 / 0975-7406.72133$. 
523 19. El-Setouhy DA, Abd El-Malak NS. Formulation of a novel tianeptine sodium 524 orodispersible film. AAPS PharmSciTech. 2010;11(3):1018-25. doi: 525 10.1208/s12249-010-9464-2.

526 20. Tayel SA, El Nabarawi MA, Amin MM, Abou Ghaly MH. Sumatriptan 527 succinate sublingual fast dissolving thin films: formulation and in vitro/in vivo 528 evaluation. Pharm Dev Technol. 2016;21(3):328-37. doi: $529 \quad 10.3109 / 10837450.2014 .1003655$.

530 21. Sagban TH, Ismail KY. Formulation and evaluation of orodispersible film of 531 sildenafil citrate. International Journal of Pharmacy and Pharmaceutical Sciences. 532 2014;6(2):81-6.

533 22. Shimoda H, Taniguchi K, Nishimura M, Matsuura K, Tsukioka T, Yamashita $534 \mathrm{H}$, et al. Preparation of a fast dissolving oral thin film containing dexamethasone: a 535 possible application to antiemesis during cancer chemotherapy. European journal of 536 pharmaceutics and biopharmaceutics : official journal of Arbeitsgemeinschaft fur 537 Pharmazeutische Verfahrenstechnik eV. 2009;73(3):361-5. doi: 538 10.1016/j.ejpb.2009.08.010.

539 23. Kumar GP, Phani AR, Prasad RG, Sanganal JS, Manali N, Gupta R, et al. 540 Polyvinylpyrrolidone oral films of enrofloxacin: film characterization and drug 541 release. Int J Pharm. 2014;471(1-2):146-52. doi: 10.1016/j.ijpharm.2014.05.033.

542 24. Dinge A, Nagarsenker M. Formulation and evaluation of fast dissolving films 543 for delivery of triclosan to the oral cavity. AAPS PharmSciTech. 2008;9(2):349-56. 544 doi: 10.1208/s12249-008-9047-7.

545 25. Li X, Kanjwal MA, Lin L, Chronakis IS. Electrospun polyvinyl-alcohol 546 nanofibers as oral fast-dissolving delivery system of caffeine and riboflavin. 547 Colloids and surfaces B, Biointerfaces. 2013;103:182-8. doi: $548 \quad 10.1016 /$ j.colsurfb.2012.10.016.

549 26. Illangakoon UE, Gill H, Shearman GC, Parhizkar M, Mahalingam S, Chatterton $550 \mathrm{NP}$, et al. Fast dissolving paracetamol/caffeine nanofibers prepared by 551 electrospinning. Int J Pharm. 2014;477(1-2):369-79. doi: $552 \quad 10.1016 / j$. .ijpharm.2014.10.036.

553 27. Tomar A, Sharma K, Chauhan NS, Mittal A, Bajaj U. Formulation and 554 evaluation of fast dissolving oral film of dicyclomine as potential route of buccal 555 delivery. International Journal of Drug Development Research. 2012;4(2):408-17. 
556 28. Susarla R, Sievens-Figueroa L, Bhakay A, Shen Y, Jerez-Rozo JI, Engen W, et 557 al. Fast drying of biocompatible polymer films loaded with poorly water-soluble 558 drug nano-particles via low temperature forced convection. Int J Pharm. 2013;455(1559 2):93-103. doi: 10.1016/j.ijpharm.2013.07.051.

560 29. Shen BD, Shen CY, Yuan XD, Bai JX, Lv QY, Xu H, et al. Development and 561 characterization of an orodispersible film containing drug nanoparticles. European 562 journal of pharmaceutics and biopharmaceutics : official journal of 563 Arbeitsgemeinschaft fur Pharmazeutische Verfahrenstechnik eV. 2013;85(3 Pt 564 B):1348-56. doi: 10.1016/j.ejpb.2013.09.019.

565 30. Sievens-Figueroa L, Bhakay A, Jerez-Rozo JI, Pandya N, Romanach RJ, 566 Michniak-Kohn B, et al. Preparation and characterization of hydroxypropyl methyl 567 cellulose films containing stable BCS Class II drug nanoparticles for pharmaceutical 568 applications. Int J Pharm. 2012;423(2):496-508. doi: 569 10.1016/j.ijpharm.2011.12.001.

570 31. Hinton C, Pratt C, De Vadetzsky E, Landwill K, McCloskey K, Schuemann H. 571 Twenty years of confectionery and chocolate progress. Twenty years of 572 confectionery chocolate progress. 1970;111.

573 32. Won DH, Kim MS, Lee S, Park JS, Hwang SJ. Improved physicochemical 574 characteristics of felodipine solid dispersion particles by supercritical anti-solvent 575 precipitation process. Int J Pharm. 2005;301(1-2):199-208. doi: $576 \quad 10.1016 / j . i j p h a r m .2005 .05 .017$.

577 33. Zimmermann A, Millqvist-Fureby A, Elema MR, Hansen T, Mullertz A, 578 Hovgaard L. Adsorption of pharmaceutical excipients onto microcrystals of 579 siramesine hydrochloride: effects on physicochemical properties. European journal 580 of pharmaceutics and biopharmaceutics : official journal of Arbeitsgemeinschaft fur 581 Pharmazeutische Verfahrenstechnik eV. 2009;71(1):109-16. doi: 582 10.1016/j.ejpb.2008.06.014.

583 34. Cho E, Cho W, Cha KH, Park J, Kim MS, Kim JS, et al. Enhanced dissolution 584 of megestrol acetate microcrystals prepared by antisolvent precipitation process 585 using hydrophilic additives. Int J Pharm. 2010;396(1-2):91-8. doi: $586 \quad 10.1016 / j . i j p h a r m .2010 .06 .016$. 
587 35. Zong X, Kim K, Fang D, Ran S, Hsiao BS, Chu B. Structure and process 588 relationship of electrospun bioabsorbable nanofiber membranes. Polymer. $589 \quad 2002 ; 43(16): 4403-12$.

590 36. Liu X, Nielsen LH, Klodzinska SN, Nielsen HM, Qu H, Christensen LP, et al. 591 Ciprofloxacin-loaded sodium alginate/poly (lactic-co-glycolic acid) electrospun 592 fibrous mats for wound healing. European journal of pharmaceutics and 593 biopharmaceutics : official journal of Arbeitsgemeinschaft fur Pharmazeutische 594 Verfahrenstechnik eV. 2018;123:42-9. doi: 10.1016/j.ejpb.2017.11.004.

595 37. Liu X, Aho J, Baldursdottir S, Bohr A, Qu H, Christensen LP, et al. The effect 596 of poly (lactic-co-glycolic) acid composition on the mechanical properties of 597 electrospun fibrous mats. Int J Pharm. 2017;529(1-2):371-80. doi: 598 10.1016/j.ijpharm.2017.06.086.

599 38. Upadhyay PP, Bond AD. Crystallization and disorder of the polytypic a 1 and 600 a 2 polymorphs of piroxicam. CrystEngComm. 2015;17(28):5266-72.

601 39. Lai F, Pini E, Corrias F, Perricci J, Manconi M, Fadda AM, et al. Formulation 602 strategy and evaluation of nanocrystal piroxicam orally disintegrating tablets 603 manufacturing by freeze-drying. Int $\mathrm{J}$ Pharm. 2014;467(1-2):27-33. doi: 604 10.1016/j.ijpharm.2014.03.047.

605 40. Borodko Y, Habas SE, Koebel M, Yang P, Frei H, Somorjai GA. Probing the 606 interaction of poly(vinylpyrrolidone) with platinum nanocrystals by UV-Raman and 607 FTIR. The journal of physical chemistry B. 2006;110(46):23052-9. doi: 608 10.1021/jp063338+.

609 41. Koland M, Sandeep V, Charyulu N. Fast dissolving sublingual films of 610 ondansetron hydrochloride: effect of additives on in vitro drug release and mucosal 611 permeation. Journal of young pharmacists: JYP. 2010;2(3):216.

612 42. Samprasit W, Akkaramongkolporn P, Ngawhirunpat T, Rojanarata T, 613 Kaomongkolgit R, Opanasopit P. Fast releasing oral electrospun PVP/CD nanofiber 614 mats of taste-masked meloxicam. Int J Pharm. 2015;487(1-2):213-22. doi: $61510.1016 / j . i j p h a r m .2015 .04 .044$.

616 43. Noyes AA, Whitney WR. The rate of solution of solid substances in their own 617 solutions. Journal of the American Chemical Society. 1897;19(12):930-4.

618 44. Vrecer F, Vrbinc M, Meden A. Characterization of piroxicam crystal 619 modifications. Int J Pharm. 2003;256(1-2):3-15. 
Dear Prof. Robert O. Williams III,

Thank you very much for the valuable feedback and forwarding the comments from the

637 reviewers. We have carefully processed the comments, and addressed their concerns. Our

638 detailed responses are listed below (in italics and green). In addition, we have also edited the

639 revised manuscript with the changes made during the revision clearly highlighted in boldface

640

641

642 Yours sincerely

643 Mingshi Yang

644

645 Reviewers' Comments:

646

647 Reviewer \#1: This manuscript presents a method for preparing ODFs using the electrospining 648 approach. The API is first crystallized into microparticles using the antisolvent method. The 
650 electrospinning. The investigation covers the physical, mechanical and performance 651 characterization of ODFs obtained using different polymeric materials as fiber-forming 652 matrices.

653 This manuscript suffers from very poor writing. It needs improvements on the flow and clarity 654 of the presentation before it gets published. Especially in the description of the methods. For 655 example, the use of stabilizers for the suspension is mentioned under the Methods section. 656 However, the specific stabilizers used are not identified until much later, in the Results and 657 Discussion section.

658 We thank the reviewer for the comments. The manuscript has been checked again and we 659 improved the flow and presentation, especially the section of Materials and Methods. The 660 information of stabilizers have been supplemented in the section of Martials and Methods:

661 "Piroxicam $(175 \mathrm{mg}$ ) was first dissolved in $2.0 \mathrm{~mL}$ of $\mathrm{N}$, $\mathrm{N}$-Dimethylformamide (DMF) to make 662 an $8.75 \%(w / v)$ drug solution. Subsequently, the solution was injected into the $13.0 \mathrm{~mL}$ distilled 663 water containing, either $0.2 \%, 0.5 \%$ or $1.0 \%(w / v)$ of each stabilizer (i.e. HPMC E15, Kollidon 664 VA64, and Pluronic F127) with a magnet-stirring rate at $1500 \mathrm{rpm}$ in a cold room $\left(4.0^{\circ} \mathrm{C}\right)$. "” 665

666 The Methods section informs the reader that "various film-forming polymers $(10 \%, \mathrm{w} / \mathrm{v})$ 667 were ..." However, it is not until 9 (nine) subsections later in the manuscript, that such film 668 forming polymers are actually identified. This is unacceptable.

669 We thank the reviewer for the comments. The film forming polymers were presented in the 670 section of Martials and Methods: "A 10\%(w/v) PVA 26-88 was prepared by dissolving 1500mg 671 of the polymer into $15 \mathrm{~mL}$ distilled water under stirring until the polymer was completely 672 dissolved. A 10\%(w/v) PVA/PVP $(2 / 1, w / w)$ was prepared by dissolving 1000mg of PVA26-88 673 and 500mg of PVP 360 into $15 \mathrm{~mL}$ distilled water under stirring until the polymers were 674 completely dissolved. Other polymer solutions containing different compositions of PVA 26$67588 /$ PVP 360 kept the total concentration at 10\% (w/v) with various mass ratios of the two 676 polymers (presented in Table 2 manuscript body). Plain ODFs were obtained by directly 677 electrospinning of the prepared polymer solution. Drug-loaded ODFs were obtained by 678 dissolving the appropriate amount of film forming polymer into PX microcrystal suspensions 679 produced with anti-solvent method, followed by electrospinning." 
681 Table 3 would be more useful if it is made more informative. I suggest to include the function

682 of the components listed (e.g., stabilizer or fiber forming polymer) on the header of the column 683 where they are listed.

684 We thank the reviewer for the comments. The Table 3 in the revised manuscript has been supplemented with the information of functionality of the excipients.

686

687 The discussion about Figure 6 should also be made clearer. It is not that the information is not 688 there, but the reader has to go back and forth between the figure and the text to get a clear idea 689 of the information provided.

690 We thank the reviewer for the comments. The discussion on Figure 6 has been improved in the 691 revised manuscript. We have updated the figure and the text in the section of the discussion.

692

693 I strongly suggest that the authors use the help of a first time reader in the preparation of their 694 revised manuscript, in order to make the improvements in clarity required.

695 We thank the reviewer for the comments. We have asked one of our colleagues to help with 696 editing the revised manuscript.

697

698 Table 5 presents the results for drug loading and entrapment efficiency. However, there is no 699 information regarding the procedures used to obtain these numbers. This type of information 700 needs to be included in the manuscript.

701 We thank the reviewer for the comments. The equation of calculation of the drug loading and 702 entrapment efficiency has been added in the revised manuscript in the in the section of Martials 703 and Methods.

704

705 There is one point that will inevitably be present in just about every reader's mind that is not 706 mentioned at all in the manuscript. It is the fate of the dimethylformamide (DMF) present in 707 the obtained films. The initial PX suspension contains about 13\% DMF. This mixture is then 708 combined with $10 \%$ polymer solution. I suppose I could go back to Table 3 and back-calculate 709 the amounts used to make the mixtures eventually subjected to electrospinning, but this is a 710 burden that the reader should not be expected to carry. The description of the methods is so 711 poor that it forces the reader to either try to figure things not provided by the authors, or to 712 brush it off altogether. The DMF content of the films is something that must be spelled loud 713 and clear. DMF has very low volatility and it is almost impossible to expect it to dry off even 
714 with the high surface are provided by the fibers. If the authors do not have information on the 715 residual content of DMF, they should at least provide the nominal content, instead of expecting 716 the reader to do those calculations. DMF is likely to have a plasticizer effect on the fibers. On 717 the other hand, the content of DMF in the ODFs is something no reader will be able to ignore 718 when looking at this manuscript.

719 We thank the reviewer for the comments. We have added the Residue solvent results in the 720 method and discussion part.

721

In the section of Materials and Method: "Residue solvent was conducted through thermogravimetric analysis (TGA) (TGA Discovery, TA Instruments, New Castle, USA). Samples around $2.5 \mathrm{mg}$ were put on platinum pans and heated from $25{ }^{\circ} \mathrm{C}$ to $250{ }^{\circ} \mathrm{C}$ at $10{ }^{\circ} \mathrm{C} / \mathrm{min}$. The obtained percentage of weight loss with temperature data was calculated as residue solvent."

727

In the section of Discussion: "DMF was used as one of the solvents for electrospun films preparation and it constituted $12.6 \%(w / v)$ in the initial PX microcrystal suspension. The obtained ODFs were slowly heated to $250^{\circ} \mathrm{C}$ and the weight loss until $180^{\circ} \mathrm{C}$ was calculated

731 for residue solvent (the boiling point of DMF is $153^{\circ} \mathrm{C}$ ). Most of the weight loss occurred 732 before the temperature reached $100{ }^{\circ} \mathrm{C}$ and the rate of weight loss with temperature became 733 slowly between $100^{\circ} \mathrm{C}$ and $160^{\circ} \mathrm{C}$. After $180^{\circ} \mathrm{C}$ a dramatic weight loss was observed, which 734 was assigned to decomposition of the chemicals. The results showed that the residue solvents 735 of the three formulations were between 3\%-4\% (Table 5) and the most solvent remained in the 736 electrospun films was more likely to be water."

Page 16, Line 294 "loaded EEFs (Fig.5)" => Spell out each acronym when first used Page 3,

740 Line 80 "gradients" => correct or clarify Page 14, Line 337 "stretches" => stretching

741 We thank the reviewer for the comments and correction. We have edited and corrected these in 742 the revised manuscript.

745 Reviewer \#3: The authors wrote a well structured manuscript. It is just needs some clarifications 746 in some points to be ready for publishing. 
749 1. In Page 3 line 78: please mention the stabilizers used.

750 We thank the reviewer for the comments. We have added the names of the stabilizers used in 751 the section of Materials and Method in the revised manuscript as follow: "Piroxicam (175 mg) 752 was first dissolved in $2.0 \mathrm{~mL}$ of $\mathrm{N}, \mathrm{N}$-Dimethylformamide (DMF) to make an $8.75 \%(\mathrm{w} / \mathrm{v}) \mathrm{drug}$ 753 solution. Subsequently, the solution was injected into $13.0 \mathrm{~mL}$ distilled water containing either $7540.2 \%, 0.5 \%$ or $1.0 \%(w / v)$ of each stabilizer (i.e. HPMC E15, Kollidon VA64, and Pluronic 755 F127) with a magnet-stirring rate at $1500 \mathrm{rpm}$ in a cold room $\left(4.0^{\circ} \mathrm{C}\right) . "$

756

757 2. In Page 3 line 85: please mention the polymers used.

758 We thank the reviewer for the comments. We have added the names of the polymers used in the 759 section of Materials and Method in the revised manuscript as follow: "A 10\%(w/v) PVA 26-88 760 was prepared by dissolving 1500mg of the polymer into $15 \mathrm{~mL}$ distilled water under stirring 761 until the polymer was completely dissolved. A 10\%(w/v) PVA/PVP $(2 / 1, w / w)$ was prepared by 762 dissolving 1000mg of PVA26-88 and 500mg of PVP 360 into 15mL distilled water under 763 stirring until the polymers were completely dissolved. Other polymer solutions containing 764 different compositions of PVA 26-88/PVP 360 kept the total concentration at 10\% (w/v) with 765 various mass ratios of the two polymers (presented in Table 2). Plain ODFs were obtained by 766 directly electrospinning of the prepared polymer solution. Drug-loaded ODFs were obtained 767 by dissolving the appropriate amount of film forming polymer into PX microcrystal suspensions 768 produced with anti-solvent method, followed by electrospinning."

770 3. In Page 4 line 103: please mention the wavelength used in UV spectrometer measurement.

771 We thank the reviewer for the comments. We have added the wavelength used in UV 772 spectrometer measurement in the section of Materials and Method: "with its absorbance at 773357 nm as $y$-axis for linear fitting."

774

775 4. It is not clear in the part of preparation using different polymers, do you add the polymer to 776 the aquoeus solution before or after the addition of piroxicam solution. 
778 We thank the reviewer for the comments. To clarify this we have revised in the section of

779 Materials and Methods: "Drug-loaded ODFs were obtained by dissolving the appropriate 780 amount of PVA 26-88/PVP 360 into PX microcrystal suspensions produced with anti-solvent method, followed by electrospinning". In addition, we moved Fig. 1 forward to make sure the clarity of preparation method.

783

784

785

786

787

788

789

790

791

792

793

794

795

796

797

798

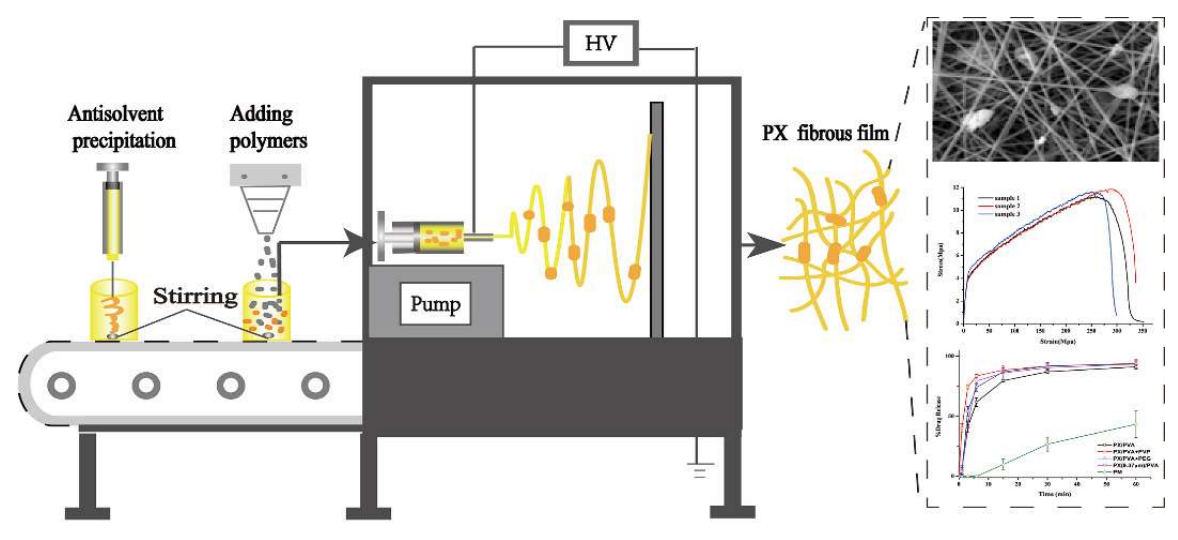

Fig.1 Preparation of PX-loaded ODFs.

5. In Page 6 line 166, What do you mean by 'The disintegration process was recorded at 60 frame/s and shown as screenshot.' Results submitted are shown in seconds.

We thank the reviewer for the comments. This sentence has been deleted and revised as follow: "The disintegration process was recorded with the video function of a smart phone and the parameter for video recording was 60fps. The disintegration process of the films were observed afterwards and screenshots were made and reported in the manuscript. The seconds in the results were the time of the screenshots were made" in the revised manuscript.

6. In Page 6 line 181, do you take the part of ODF according to its weight or dimension.

We thank the reviewer for the comments. We took part of the ODFs according to its dimension. Meanwhile but we have measured their thickness before cutting. As shown in the table below, their thickness were similar, and their weights were also similar. We have commented on the thickness and weight of the films in the revised manuscript.

\begin{tabular}{ll}
\hline Formulation & Thickness Weight $(\mathrm{mg})$ \\
& $(\mu \mathrm{m})$
\end{tabular}




\begin{tabular}{lcc}
\hline PX/PVA & $43.0 \pm 4.0$ & $11.5 \pm 0.2$ \\
PX/PVA+PVP & $45.0 \pm 1.0$ & $12.8 \pm 0.1$ \\
PX/PVA+PEG & $46.0 \pm 3.1$ & $12.5 \pm 0.4$ \\
\hline
\end{tabular}

799

800

801

802

803

804

805

806

807

808

809

810

811

812

813

814

815

816

817

818

819

820

821

822

823

824

825

826

827

7. Why did you choose distilled water as dissolution medium. The film is supposed to disintegrate in the mouth (simulated salive solution) and also as you said in page 17 line 383 'disintegrate or dissolve fast in mouth for ease of swallowing, and the dissolved PX was intended to be absorbed through gastrointestinal tract.' I think dissolution should be repeated in simulated saliva solution.

We thank the reviewer for the comments. We chose distilled water based on the following considerations. Due to fast dissolving of film forming polymer, ODFs will disintegrate fairly quick and then the microcrystal suspension would be swallowed. The dissolution of PX in simulated saliva solution (rather than gastric media) would not be relevant. Dissolution was a rough experiment to reflect the in-vitro dissolution rate of the drug in the formulations. No dissolution medium (even bio-relevant dissolution medium) would realize the real dissolution of the formulations in vivo. We chose distilled water as it is an easy and efficient dissolution medium, which could also be reasonable medium to investigate and reflect the in-vitro dissolution profile of $P X$. It also has been used as dissolution media for PX dissolution before (Lai F et al.2014). In addition, we chose water to see whether fiberous electrospun ODFs would have any influence on the PX dissolution, a poorly water-soluble drug.

Lai F, Pini E, Corrias F, et al. Formulation strategy and evaluation of nanocrystal piroxicam orally disintegrating tablets manufacturing by freeze-drying[J]. International journal of pharmaceutics, 2014, 467(1-2): 27-33.

8. It is clear in page 9 that you tried different ratios of PVA/PVP and PVA alone was the worst formulation. Then after adding PX, you did not make use of your result, you tried effect of PEG on PVA alone (it is clear to be the worst formulation) and not on the best formula. Please clarify your point of view of comparing with PVA while your screening results showed it is the worst formulation. Also you continued on 2:1 PVA:PVP and not 1:1 ratio, how did you discriminate your results. 
828 We thank the reviewer for the comments. Firstly, It has been reported that PEG could act as a 829 plasticizer to facilitate formation of casting film and facilitate disintegration. Therefore, we 830 added PEG to PVA film to see whether PEG would facilitate the disintegration of PVA films 831 obtained from electrospinning. Secondly, PVA/PVP (2:1/w:w) films exhibited very fast 832 disintegration in the test (less than 5 seconds). It would not be easy to see the influence of PEG 833 on ODFs if we add PEG into PVA/PVP (2:1/w:w) films. In fact, we tried to add PEG into 834 PVA/PVP (2:1/w:w) film formulations (data not shown). However, the electrospinning process 835 became unstable. Therefore, we did not continue with this formulation.

836 As for PVA/PVP (1:1/w:w), we did not continue this formulation because the electrospun films 837 became more soft with an increase in the proportion of PVP. In addition, the film became more 838 hygroscopic with an increase in the proportion of PVP. In the disintegration tests with PX 839 loaded electrospun PVA/PVP (2:1/w:w) film, the disintegration of the films was ca. 2 seconds.

840 We postulated that PVA/PVP (1:1/w:w) film could be faster. However, the difference might not 841 be obvious. Lastly, we observed that the electrospinning process of PVA/PVP (2:1/w:w) was 842 more stable as compared to that of 1:1. Beads could be observed with an increase in PVP in 843 the formulation (Table1. Fig.1 below).

844 Table 1. Experimental observation of electrospinning of PVA and PVP binary mixture solutions

\begin{tabular}{llll}
\hline PVA/PVP mass ratio & Electrospinnablity & Beads & Process stability \\
\hline $4: 1$ & Yes & Uniform & Yes \\
$2: 1$ & Yes & Uniform & Yes \\
$1: 1$ & Yes & + & No \\
$1: 2$ & Yes & ++ & No \\
\hline
\end{tabular}

845 

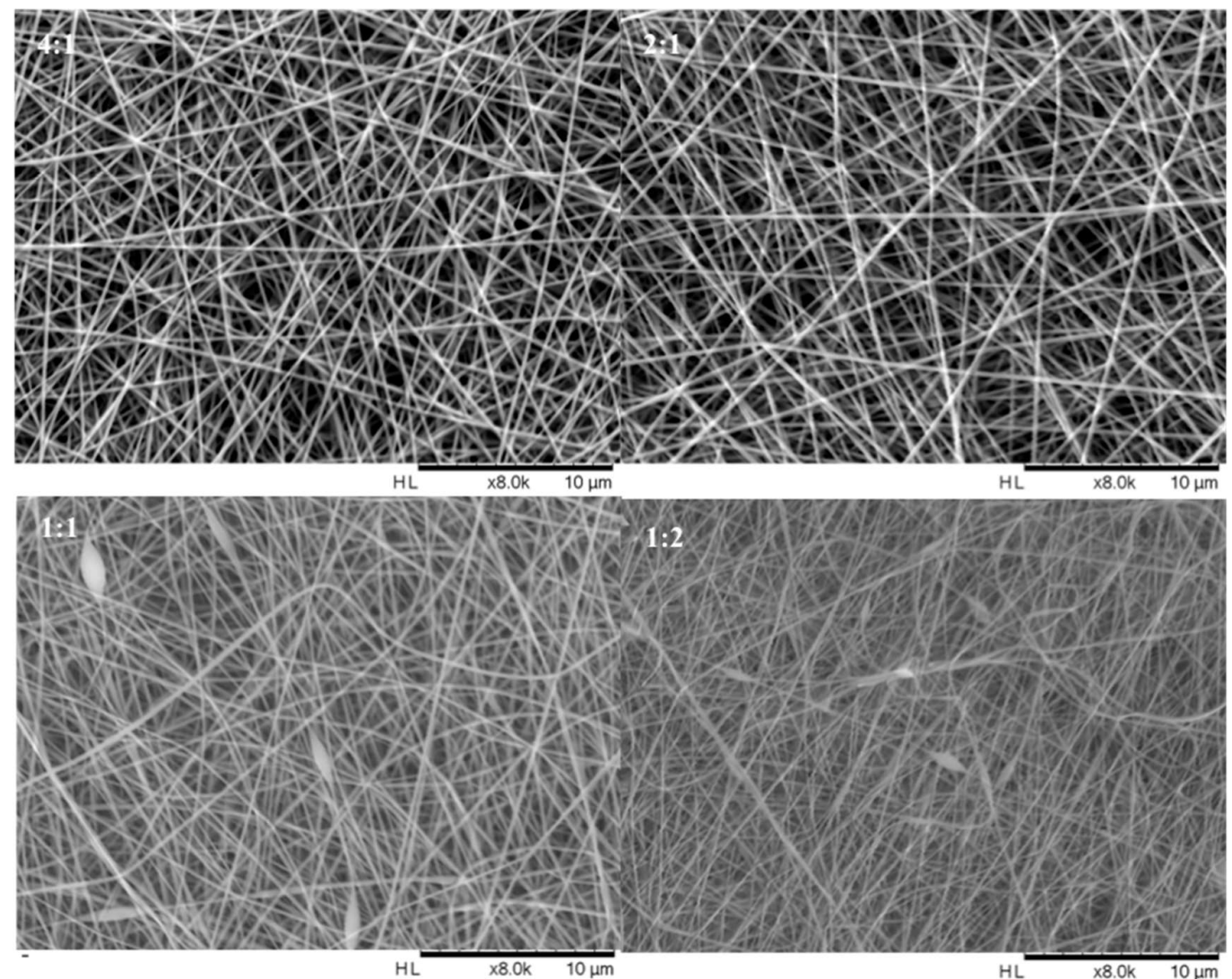

Fig. 1 SEM images of electrospun films composed of PVA and PVP at mass ratios of 4:1; 2:1, 1:1, and $1: 2$.

The changes in the revised manuscript:

851 Page 2, Line 53. Adding sentence "The stabilizers and their optimal concentrations for 852 precipitating PX in anti-solvent precipitation method were selected among poloxamer, 853 copovidone and hydroxypropyl methylcellulose at three different concentrations, respectively."

855 Page 3, Line 78.Adding sentence "PX microcrystal suspensions were prepared with anti-solvent 856 precipitation method." 
859 E15, Kollidon VA64, and Pluronic F127) with a magnet-stirring rate at $1500 \mathrm{rpm}$ in a cold $\operatorname{room}\left(4.0^{\circ} \mathrm{C}\right) . "$

861

862 Page 3, Line 97. Adding sentence “A 10\%(w/v) PVA 26-88 was prepared by dissolving $8631500 \mathrm{mg}$ of the polymer into $15 \mathrm{~mL}$ distilled water under stirring until the polymer was 864 completely dissolved. A 10\%(w/v) PVA/PVP (2/1;w/w) was prepared by dissolving $1000 \mathrm{mg}$ 865 of PVA26-88 and 500mg of PVP 360 into $15 \mathrm{~mL}$ distilled water under stirring until the polymer 866 was completely dissolved. Other polymer solutions containing different compositions of PVA

867 26-88/PVP $360 \mathrm{kept}$ the total polymer concentration at $10 \%(\mathrm{w} / \mathrm{v})$ with various mass ratios of 868 the two polymers (Table 2). Plain ODFs were obtained by directly electrospinning the prepared

869 polymer solutions. Drug-loaded ODFs were obtained by dissolving the appropriate amount of 870 PVA 26-88/PVP 360 into PX microcrystal suspensions produced with anti-solvent method, 871 followed by electrospinning."

872

873 Page 6, Line 173. Adding sentence "The disintegration process was recorded with the video 874 function of a smart phone and the parameter for video recording was $60 \mathrm{fps}$. The disintegration 875 process of the film was observed afterwards and screenshots were made and reported. The 876 seconds in the results were the time of the screenshots was made."

877

878 879 880 881

882 "Residue solvent was conducted through thermogravimetric analysis (TGA) (TGA Discovery, 883 TA Instruments, New Castle, USA). Samples around $2.5 \mathrm{mg}$ were put on platinum pans and 
884 heated from $25{ }^{\circ} \mathrm{C}$ to $250{ }^{\circ} \mathrm{C}$ at $10{ }^{\circ} \mathrm{C} / \mathrm{min}$. The obtained percentage of weight loss with

885 temperature data was calculated as residue solvent."

886

887 Page 10, Line 263. Adding function of the components in table 5.

888

889

Page 13, Line 314. Updating with "electrospun ODFs"

890

891 Page 14, Line 341. Adding sentence "These indicated that PX transformed from Form I to

892 monohydrate form after anti-solvent precipitation. The physical mixtures were prepared with

893 monohydrate form PX and the polymers in the same ratios as in the drug-loaded ODFs.

894 Comparing the XRPD patterns of the physical mixtures with their corresponding formulation

895 diffractgrams, PX microparticles kept their monohydrate form, which suggested that the

896 electrospinning process did not distort the solid state of PX microparticles."

897

898 Page 15, Line 360 Updating with stretching.

899

900 Page 18, Line 409. Adding sentence "DMF was used as one of the solvents for electrospun

901 films preparation and it constituted $12.6 \%(\mathrm{w} / \mathrm{v})$ in the initial PX microcrystal suspension. The

902 obtained ODFs were slowly heated to $250{ }^{\circ} \mathrm{C}$ and the weight loss until $180{ }^{\circ} \mathrm{C}$ was calculated

903 for residue solvent (the boiling point of DMF is $153{ }^{\circ} \mathrm{C}$ ). Most of the weight loss occurred

904 before the temperature reached $100{ }^{\circ} \mathrm{C}$ and the rate of weight loss with temperature became

905 slowly between $100^{\circ} \mathrm{C}$ and $160^{\circ} \mathrm{C}$. After $180^{\circ} \mathrm{C}$, a dramatic weight loss was observed, which

906 was assigned to decomposition of the chemicals. The results showed that the residue solvents

907 of the three formulations were between 3\%-4\% (Table 5)."

908 
909 Page 18, Line 427. Adding sentence "Thet hicknesses of the film cuts for dissolution study

910 were measured and the corresponding weights of the films were presented in Table 5."

911 